\title{
Pensar e "inscribir" mientras se investiga: un estudio de caso
}

\author{
Merce Garcia-Mila ${ }^{1}$, Christopher Andersen ${ }^{2}$ \\ Y NuBIA E. ROJO ${ }^{1}$ \\ ${ }^{1}$ Universidad de Barcelona; ${ }^{2}$ Obio State University

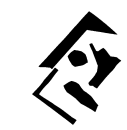 \\ Resumen
}

Las inscripciones sobre el mundo a través de dibujos, notaciones, tablas, diagramas, etc. son una parte esencial de toda investigación científica (Latour y Woolgar, 1979). Latour define "inscripción" como medio a través del cual las propiedades del mundo pueden fijarse, lo que permitiría centrarse en aquellos aspectos esenciales para construir conocimiento. Por otro lado, la investigación científica tiene lugar mediante procesos que implican el planteamiento de preguntas e hipótesis, el diseño de experimentos, hacer predicciones, observaciones y mediciones, registrar datos y interpretar resultados, consultar y analizar el registro de los datos, verificar y reaccionar ante los datos discrepantes, elaborar argumentos, construir varias representaciones de los datos y coordinarlas con teorías previas (Duschl et al, 2007). Este trabajo tiene como objetivo el análisis de la regulación mutua entre las prácticas inscripcionales y la investigación científica en alumnos de final de enseñanza primaria. Esta relación la ilustramos mediante un análisis microgenético de estudio de caso.

Palabras clave: Investigación científica, estudio de caso, toma de notas, metacognición, preadolescentes, función epistémica.

\section{Thinking and "inscribing" while investigating: A case study}

\begin{abstract}
Inscriptions of the world by means of drawings, notations, diagrams, etc. are an essential part of any scientific research (Latour E Woolgar, 1979). Latour defines "inscription" as a means to fix the properties of the world, so that the essential features of the world can be captured to build knowledge. On the other hand, scientific inquiry involves processes like asking questions, experimental design, making predictions, measuring and observing, registering data, and interpreting results (either confirming or disconfirming), reviewing notes, and verifying data to build arguments and to reformulate prior theories (Duschl et al, 2007). The present study has the goal of analysing the mutual interaction between inscriptional practices and scientific inquiry among primary school students (6th graders). We illustrate this relationship by means of a microgenetic case study.

Keywords: Scientific inquiry, case study, note-recording, metacognition, preadolescents, epistemic function.
\end{abstract}

Agradecimientos: El presente trabajo ha sido posible gracias a la financiación de los Proyectos SEJ2006-15639C02-02; PR2008-0155 por el Ministerio de Ciencia y Tecnología Español; así como del Proyecto NSF Award Number DMR-0820414 por la National Science Foundation (US). También queremos agradecer de manera especial a los alumnos de la Escuela Piaget de Barcelona su participación en la investigación cuyos datos se presentan en este artículo. Sin su participación esta investigación no habría sido posible.

Correspondencia con los autores: Merce Garcia-Mila. Universidad de Barcelona. Passeig de la Vall d'Hebron, 171. 08035 Barcelona. Tel.: 34 933125833.E-mail: mgarciamila@ub.edu 
I spent a lot of time on writing, but usually while the work was going on. And I find in general only when one tries to write it up, then do I find the gaps. I cannot complete a piece of work and then sit down and write the paper (Holmes, 1987, p. 226).

La cita anterior corresponde al testimonio directo de la conversación entre Holmes y Krebs acerca de la redacción del artículo de este último en 1932 sobre su descubrimiento del ciclo de la urea. Las palabras de Krebs ilustran claramente el papel epistémico de la escritura en el desarrollo de teorías durante la investigación científica y nos acercan al objetivo del presente artículo: analizar la misma relación en alumnos cuyas estrategias de investigación están emergiendo.

La idea de Latour sobre el papel de las inscripciones en el trabajo empírico (Latour y Woolgar, 1979) nos sirve de punto de partida para fundamentar nuestro planteamiento teórico. La noción de "inscripción" se refiere a representaciones geométricas, mapas, diagramas, gráficos, tablas, notación química, notaciones algebraicas, escritura, notaciones numéricas, etcétera, que se utilizan para representar aspectos del mundo. Serían el medio a través del cual las propiedades del mundo se pueden fijar, lo que permitiría centrarse en aquellos aspectos esenciales para construir conocimiento (Latour, 1990). De acuerdo con diversos autores entendemos que representar externamente no se reduce a copiar lo que uno ve sino que implica inventar o adaptar convenciones de un sistema representacional para, por ejemplo, seleccionar, magnificar, fijar, componer, o registrar para construir un argumento (Lehrer y Schauble, 2006; Olson, 1994).

Los resultados fruto del reciente interés teórico por la relación entre las representaciones externas y la cognición (Olson, 1994; Wells, 1999), así como de estudios en historia de la ciencia que analizan los cuadernos de laboratorio de los científicos y el desarrollo de la ciencia nos llevan a destacar tres importantes funciones de las representaciones externas en el avance conceptual más allá de la función comunicativa. La primera se refiere a la función mnemónica en tanto que necesidad de un registro detallado de lo que se piensa para evitar el olvido. Tweney en el estudio sobre Faraday (1991, p. 305), demuestra la gran preocupación de este científico por "anotar para prevenir el cambio en lo que se recuerda". La segunda se refiere a la función organizativa, la cual permitiría el tratamiento de la información, su organización y su estructuración con el fin de objetivarla y tomar conciencia de relaciones difícilmente visibles de otra manera (Wells, 1999). Finalmente, la tercera se refiere a la función epistémica. La ciencia se realiza creando, usando, manipulando y transformando inscripciones en tanto que objetos semióticos (Lemke, 2002). La función epistémica está muy bien ilustrada en el análisis que Gruber (1974) hace del trabajo de Darwin. Este análisis muestra la adaptación mutua entre la representación interna y la representación externa a través de la revisión de Darwin de sucesivas representaciones externas (diagrama de árbol) y la génesis de su idea revolucionaria (el eslabón perdido entre primates y humanos) en la teoría de la evolución.

Muy relevante para nuestra investigación es el trabajo de Klein (2000) en el que se pregunta si este análisis histórico se puede extrapolar, momento-a-momento, a la manera de investigar, razonar y argumentar de nuestros alumnos. Es decir, ¿pueden los efectos observados a nivel histórico ser aplicados a un nivel microgenético? Esta es la pregunta que nos planteamos en la presente investigación. En los últimos años ha habido un interés creciente por el análisis de la relación entre las inscripciones y el desarrollo conceptual por un lado (Klein, 2000; Lehrer, Schauble, Carpenter y Penner, 2000); y por otro lado, entre éstas y el desarrollo de las estrategias de investigación científica (Eberbach y Crowley, 2009; Kanari y Millar, 2004; Klaczynski, 2000; Masnick y Klahr, 2003; Wu y Krajcik, 2006). En este último grupo es donde se enmarca nuestro trabajo (Garcia-Mila y Andersen, 2007; Garcia-Mila, Andersen y Rojo, 2009). 
Para poder describir la relación entre prácticas inscripcionales y la investigación científica es necesario definir qué entendemos por esta última. Según Duschl, Schweingruber y Shouse (2007), los procesos de investigación científica tienen lugar mediante decisiones acerca de lo que llama la atención, lo que se registra, se organiza y se transforma en datos para su óptima visualización y así posteriormente, poder transformarse en teorías a partir de inferencias. Estos procesos se engloban en el ciclo completo de la investigación científica: planteamiento de preguntas e hipótesis, el diseño de experimentos, hacer preediciones, observaciones y mediciones, registrar datos y interpretar resultados, consultar y analizar el registro de los datos, verificar y reaccionar ante los datos discrepantes, elaborar argumentos, construir varias representaciones de los datos y coordinarlas con teorías previas.

El análisis de los procesos anteriores tiene una larga tradición en psicología cognitiva (ver Zimmerman, 2000, 2005, para una revisión), aunque tradicionalmente estas investigaciones se han centrado, o bien en las estrategias de diseño experimental y recogida de datos, o en las estrategias de interpretación de datos y evidencia para generar conclusiones, es decir, estrategias inferenciales. Sus resultados muestran los sesgos que aparecen en el razonamiento cuando los alumnos presentan teorías previas. Por un lado, las teorías previas afectan la forma en que se estructura y organiza el problema y sesgan las decisiones sobre qué aspectos son importantes y qué datos se deben de recoger (Cheng y Novick, 1992; Klahr, 2000; Kuhn, Garcia-Mila, Zohar y Andersen, 1995). Por otro lado, las expectativas están estrechamente relacionadas con la observación y la interpretación de los datos. Se tiende a ignorar aquellos datos que contradicen las propias expectativas teóricas, o incluso se interpreta la evidencia parcialmente. Se reconocen sólo aquellos resultados que confirman las propias expectativas, utilizando a menudo distintos patrones de evaluación en función de si los datos confirman o refutan las teorías previas; o incluso, es común que se distorsionen los datos para encajar las teorías (Eberbach y Crowley, 2009; Kuhn et al., 1995; Zimmerman, 2000).

Los sesgos anteriores se observan claramente en preadolescentes, aunque también están presentes en adultos. Estudios microgenéticos han mostrado como en todos ellos se observa una alta variabilidad intrasujeto (Kuhn et al., 1995; Schauble, 1990). A todas las edades los individuos muestran una coexistencia de estrategias, que van desde menos a más efectivas. A lo largo de diversos encuentros con la tarea cambia la frecuencia de uso de las estrategias, con una caída en la frecuencia de las menos efectivas y un aumento lento pero progresivo de las más efectivas. Estamos de acuerdo con Kuhn (2002) cuando interpreta esta variabilidad intrasujeto a partir de la metacognición. Desde esta perspectiva, la metacognición se concreta en tres componentes: (1) el componente metatarea, el cual consiste en la comprensión de la tarea, así como de los medios para resolverla: (2) el componente metaestratégico, entendido como la compresión del porqué se utiliza una estrategia determinada frente a simplemente ser capaz de utilizarla; y finalmente, (3) el componente metacognitivo definido como la conciencia de poner un paréntesis y aislar las expectativas o teorías previas y contemplar alternativas que deben ser sometidas a prueba. Estos tres componentes de la metacognición serán fundamentales en el análisis del presente trabajo para interpretar la relación entre prácticas inscripcionales y el razonamiento implicado en la investigación científica.

A pesar de la importancia de las prácticas inscripcionales en los procesos de investigación, trabajos previos muestran que los alumnos no utilizan registros escritos de manera espontánea cuando investigan, ni tampoco revisan sus notas a pesar de que cuando lo hacen, sus prácticas mejoran (Eberbach y Crowley, 2009; Garcia-Mila y Andersen, 2007; Klein, 2000). Keys, Hand, Prain y Collins 
(1999) por ejemplo, observó que los adolescentes no relacionaban sus observaciones con nuevas hipótesis, lo que se podía atribuir al hecho de que las observaciones eran escasas, incompletas y por tanto poco relevantes. Por otro lado, Ford (2005) muestra cómo alumnos de $3 \mathrm{r}$ curso anotaban descripciones rigurosamente detalladas de muestras minerales pero hacían muy pocas asociaciones entre sus observaciones y los conceptos relacionados. Las dos investigaciones anteriores ponen de manifiesto los dos características fundamentales para una buena toma de notas en el laboratorio: completas y económicas. Estos criterios muestran la tensión planteada por Karmiloff-Smith (1979) en un estudio microgenético sobre el uso de notaciones para recordar. Las notas deben incluir suficiente información de forma que permitan recordar los elementos críticos más tarde sin tener que repetir el trabajo experimental. A la vez, deben evitar la redundancia que interferiría con el uso de la información en futuras investigaciones.

Tal como hemos planteado en otro lugar (Garcia-Mila y Andersen, 2007), para que el individuo se beneficie de las inscripciones de cualquier tipo, debe ser consciente de la ayuda que éstas aportan, y por tanto, ver la necesidad de anotar en ciertas tareas. El individuo debe ser capaz de distinguir una buena de una mala inscripción, y ser consciente de las características que hacen que una inscripción sea útil, y aplicarlo a la toma de notas.

Este trabajo plantea tres objetivos. El primero, mostrar la relación entre la práctica inscripcional y las estrategias de investigación científica. El segundo objetivo consiste en apoyar la relación anterior con un análisis microgenético de la regulación mutua. Y finalmente el tercer objetivo consiste en plantear las características que debe presentar el diseño de la tarea para promover las prácticas inscripcionales mientras se investiga.

\section{MÉTODO}

\section{Participantes}

Aunque la muestra del estudio consistió en 34 alumnos de $6^{\circ}$ curso de enseñanza primaria de una escuela pública de Barcelona (media: 11;6 y rango: 11;0$13 ; 0)$, en el presente trabajo trataremos los resultados de un alumno de la muestra (David), apoyándonos cuando sea necesario de datos cuantitativos publicados en Garcia-Mila et al. (2009). Todos los alumnos participaron individualmente en dos sesiones semanales de 45 minutos de duración durante 7 sesiones. David es un participante representativo respecto a la aplicación de estrategias de investigación científica, aunque pertenece al grupo de participantes cuyas notas mostraron mayor estructuración y organización.

\section{Procedimiento}

Se pidió a los participantes que investigaran los factores que hacían crecer una planta cuyo ciclo se termina en 15 días (Wisconsin Fastplants). Se acotó la investigación a tres factores: tipo de luz (natural o artificial); tipo de abono (natural o ecológico) y tipo de semilla (Rosette o Brassica). Las sesiones consistían en trabajo autodirigido de investigación y se desarrollaban mediante entrevistas individualizadas en las que se formulaban preguntas del tipo: ¿Qué pretendes averiguar con este experimento? ¿Cuál es tu predicción para este experimento? ¿Que bas descubierto? ¿Cómo sabes que ... es mejor que ...? En la primera sesión se proporcionó una libreta de laboratorio a los alumnos y se les pidió que pusieran su nombre, pues sería para su uso exclusivo y que estaría allí para cuando la necesitasen. También se proporcionó el material para la investigación (macetas, tierra, abonos, semillas, etiquetas adhesivas para escribir sus nombres, fechas, etcétera). Los alumnos tra- 
bajaron durante 7 sesiones. En la $1^{\mathrm{a}}, 2^{\mathrm{a}}$ y $4^{\mathrm{a}}$ plantaron las semillas. A partir de la $2^{\mathrm{a}}$ ya pudieron observar el crecimiento de las plantas. En las sesiones $3^{\mathrm{a}}$ y $7^{\mathrm{a}}$ se pidió a los alumnos que escribieran un informe sobre lo que habían descubierto. La tabla I muestra la secuencia de actividades a lo largo de las 7 sesiones (ver Tabla I).

TABLA I

Secuencia de Sesiones para la Tarea Experimental

\begin{tabular}{|c|c|c|c|}
\hline Tiempo & Sesión & Actividad Investigadora & Inscripción \\
\hline \multirow[t]{2}{*}{ Semana 1} & Sesión 1 & $\begin{array}{l}\text { Introducción. Evaluación de Teorías } \\
\text { Diseño experimentos } 1,2,3,4\end{array}$ & Toma de notas \\
\hline & Sesión 2 & $\begin{array}{l}\text { Observación de Datos Exp.: 1,2,3,4 } \\
\text { Diseño experimentos: } 5,6,7,8\end{array}$ & Toma de notas \\
\hline \multirow[t]{2}{*}{ Semana 2} & Sesión 3 & Observación de Datos Exp.: 1,2,3,4,5,6,7,8 & $\begin{array}{l}\text { Toma de notas } \\
\text { Informe } 1\end{array}$ \\
\hline & Sesión 4 & $\begin{array}{l}\text { Observación de Datos } 1,2,3,4,5,6,7,8 \\
\text { Diseño experimentos: } 9 \text { y } 10\end{array}$ & Toma de notas \\
\hline \multirow[t]{2}{*}{ Semana 3} & Sesión 5 & $\begin{array}{l}\text { Observación de Datos Exp.: 1,2,3,4,5,6,7,8,9,10 } \\
\text { Eliminación Exp.:1,2,3, } 4 \text { de la Sesión } 1\end{array}$ & Toma de notas \\
\hline & Sesión 6 & $\begin{array}{l}\text { Observación de Datos Exp.: 5,6,7,8, 9,10 } \\
\text { Eliminación Exp.: 5,6,7,8 de la Sesión } 2\end{array}$ & Toma de notas \\
\hline Semana 4 & Sesión 7 & $\begin{array}{l}\text { Observación de Datos Exp.: 9, } 10 \\
\text { Conclusiones } \\
\text { Evaluación de Teorías }\end{array}$ & $\begin{array}{l}\text { Toma de notas } \\
\text { Informe } 2\end{array}$ \\
\hline
\end{tabular}

\section{Tarea}

Un valor importante de esta investigación se encuentra en el hecho de que se pidió a los participantes realizar el ciclo completo de la investigación: diseño experimental y análisis de los datos para hacer inferencias y llegar a conclusiones de manera repetida de forma que el feedback de los resultados de los experimentos podía actuar de ayuda a la reflexión sobre la eficacia estratégica y su mejora. Esta característica permite analizar las estrategias de las distintas fases desde el punto de vista de su interacción mutua. La tarea se presentó de la manera siguiente

Nuestro gobierno quiere probar una semilla (Brassica) que ya ba sido probada previamente en otro país con resultados excelentes para alimentar el ganado. Además el Gobierno tiene otra semilla (Rosette), muy similar a Brassica, que podría ser tan eficaz como ella, y que además es mucho más barata. Tu tarea consiste en determinar las condiciones en las que la planta producirá una mejor cosecha. Deberás decidir cuál de las dos semillas da un mejor resultado, así como decidir si es mejor utilizar un fertilizante químico o ecológico y si es mejor la luz natural del sol o la luz artificial.

En la tabla II se puede observar un resumen de los efectos de las tres variables: tipo de semilla: Brassica (B) o Rosette (R), tipo de luz; Natural (N) o Artificial (A), y tipo de abono: Ecológico (E) o Químico (Q).

TABLA II

Efectos de las Variables sobre el Crecimiento de las Plantas

\begin{tabular}{ll}
\hline Variable & Efecto \\
\hline Tipo de semilla (Brassica-B vs. Rosette-R) & Brassica > Rosette \\
Tipo de abono (Ecológico-E vs. Químico-Q) & Ecológico $>$ Químico \\
Tipo de luz (Natural-N vs. Artificial-A) & Artificial $>$ Natural \\
\hline
\end{tabular}




\section{RESULTADOS}

Tal como hemos mencionado, los resultados consisten en una análisis microgenético de uno de los participantes. El análisis se apoyará con resultados cuantitativos ya publicados (Garcia-Mila et al., 2009). El análisis sigue un recorrido de la actividad del participante. Este recorrido coincide con la propia estructura de la tarea y permite centrarnos primero en las estrategias de diseño experimental, y en segundo lugar, en las estrategias inferenciales. Es importante señalar que las estrategias no son independientes y que el enfoque será global cuando el análisis así lo requiera. Los dos bloques en los que se estructuran los resultados integran en su análisis las prácticas inscripcionales.

\section{Estrategias de Diseño Experimental: recogida de datos}

Estrategias de combinación factorial de variables

Antes de empezar a investigar se pidió a David que explicitara sus teorías previas. David explicó que: Brassica era mejor ya que ya habia sido probada en otro país y había funcionado bien. También explicó que: la luz natural era mejor que la artificial, porque era natural, provenía del sol. También aplicó su teoría de "lo natural mejor" al abono y dijo: el ecológico será mejor, porque es natural, no tiene productos químicos, porque proviene de la tierra. Vemos por tanto que David sigue las previsiones hechas por los experimentadores de inducir con el planteamiento de las variables que tanto la Brassica, como la luz natural y el abono ecológico serian los niveles de las variables que se hipotetizarían como mejores que sus niveles contrarios (Rosette, luz artificial y abono químico). De sus teorías previas, David debe refutar su teoría que defiende que la luz natural es mejor que la artificial.

En la sesión 1 David diseña los cuatro primeros experimentos: BAE (Brassica, luz artificial y abono ecológico), RNQ (Rosette, luz natural y abono químico), BNE (Brassica, luz natural y abono ecológico), y finalmente, RAQ (Rosette, luz artificial y abono químico). Cuando se le pregunta lo que quiere averiguar, éste contesta que quiere ver cuál crece más. La estrategia utilizada por David es muy común: diseñar dos pares de experimentos con todos los contrarios. Esta estrategia es lo que Tschirgi (1980) denomina CA strategy (Change-All) como un paso previo a la estrategia de control de variables, que esta autora denomina VOTAT strategy (Vary-One-Thing-At-a-Time). Por parte de David no observamos ningún indicio de control de variables explícito, aunque podemos constatar que los 4 experimentos diseñados constituyen dos pares en los que la variable luz está controlada. Una vez que David acabó con el diseño de experimentos, escribió las etiquetas en las macetas y preparó una hoja de datos muy primitiva en su libreta de laboratorio para rellenar sus observaciones (ver Figura 1).

David estructuró la hoja de la libreta en tres partes, cada una correspondiente a cada una de las tres sesiones de recogida de datos. Debajo de los apartados 1 y 2 puso 4 números, uno para cada uno de los experimentos, sin explicitar las variables. En todas las sesiones a partir de la primera, se empezaba observando el crecimiento de los experimentos preparados durante las sesiones anteriores. Las plantas de David casi no habían crecido, por tanto dijo que no podía decir nada y se centró en sus notas. David numeró las macetas de la $1^{\text {a }}$ sesión, del 1 al 4, así como las de la $2^{a}$ sesión, también del 1 al 4 . Cuando procedió a registrar sus observaciones de la sesión 2 , se dio cuenta de que la forma en que había estructurado la hoja de su libreta no era apropiada para anotar sus registros. Vemos aquí un proceso autorregulador que implica comprensión de la demanda de la tarea y conciencia de lo que se va a necesitar para poder resolverla, y a su vez, conciencia del poder estructurador de un registro bien hecho. David decidió empezar de 
FIGURA 1

Primera página de la Libreta

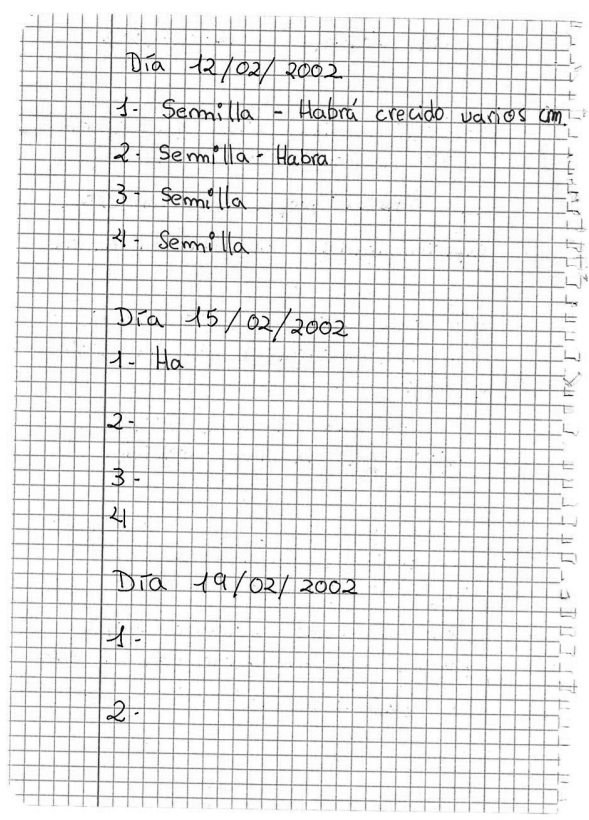

nuevo en otra hoja de la libreta, estructurando las dos hojas siguientes en 4 partes, una para cada experimento diseñado en la primera sesión. Las figuras 2 y 3 muestran las primeras hojas en las que se ven registrados datos de las dos primeras sesiones.

FIGURA 2

Segunda página de la Libreta

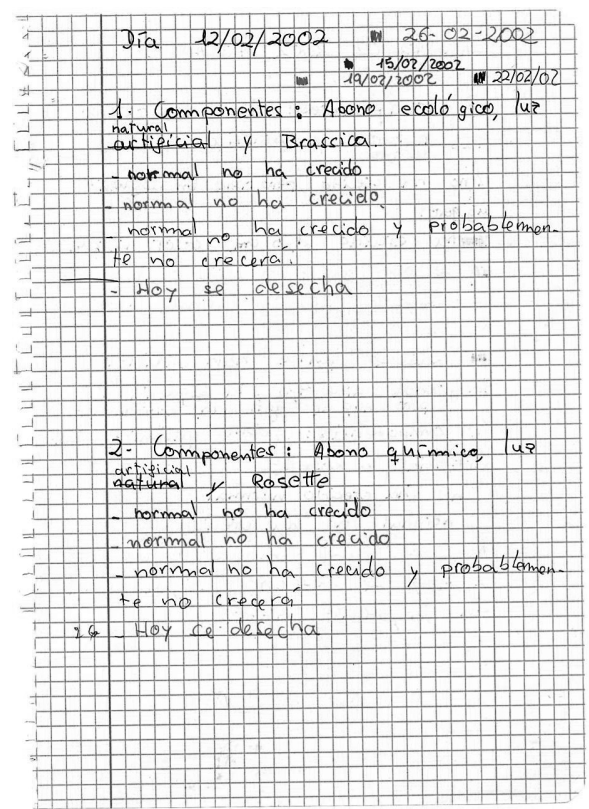


FIGURA 3

Cuarta página de la Libreta

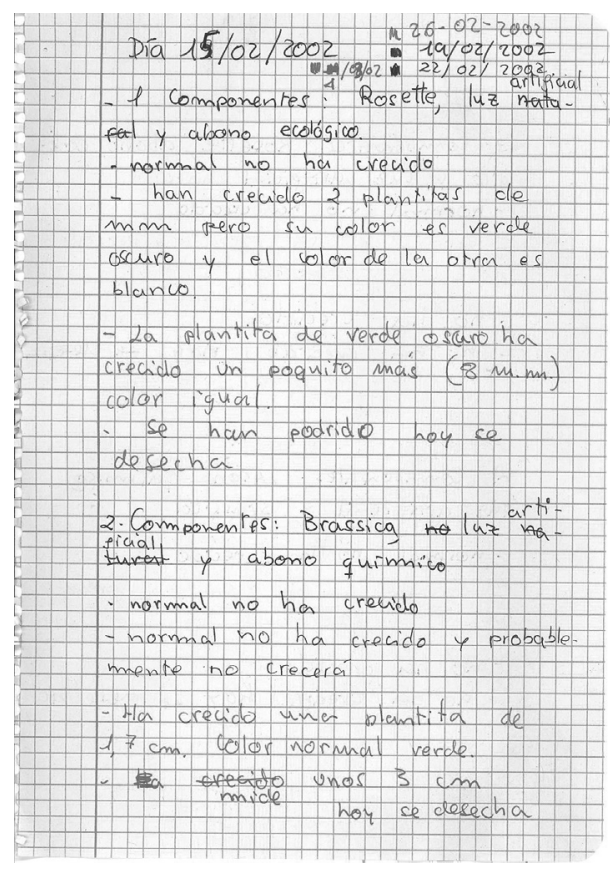

Posteriormente, David añadió las observaciones de la sesión 2 en tinta de color azul. David utilizó el código de colores para poder identificar la planta según la fecha (un color distinto para cada día). Aunque definió muy rigurosamente el significado del código de los colores mediante una leyenda, cambió el código de colores en la siguiente sesión. A partir de esta organización, David pudo economizar enormemente el registro de las observaciones, además de añadir un factor estructurador, permitiéndole observar los patrones de crecimiento mejor.

Cuando en la sesión 2 se le preguntó a David qué experimentos quería diseñar, se dio cuenta de que tenía que organizarse con el fin de ser ordenado y eficiente y preguntó:

¿Puedo escribir mis planes en la libreta? Un cuadro con todas las posibilidades de abono químico, entre Brassica y Rosette, y con luz natural o artificial, un diagrama?

Aquí es cuando David tomó conciencia de la demanda de la tarea, lo que Kuhn (2002) denomina componente metatarea de la cognición. Podemos ver el diagrama que David creó en la última página de su libreta para organizar su recogida de datos (Figura 4). El cuadro es un intento de representar todas las combinaciones posibles y a pesar de que el diagrama sólo combina dos factores, y que le faltaría una línea para poder marcar todas las distintas combinaciones de factores ${ }^{1}$, es realmente una ayuda semiótica importante. Vemos además que debajo del diagrama, David hace la traducción en las ocho combinaciones marcando con una cruz en rojo las combinaciones correspondientes a los experimentos ya diseñados en la sesión 1 y con una cruz en negro los que debería diseñar en la segunda sesión, añadiendo la leyenda explicativa en el margen. 
FigURA 4

Ùltima página de la Libreta. diagrama para la Combinación Factorial

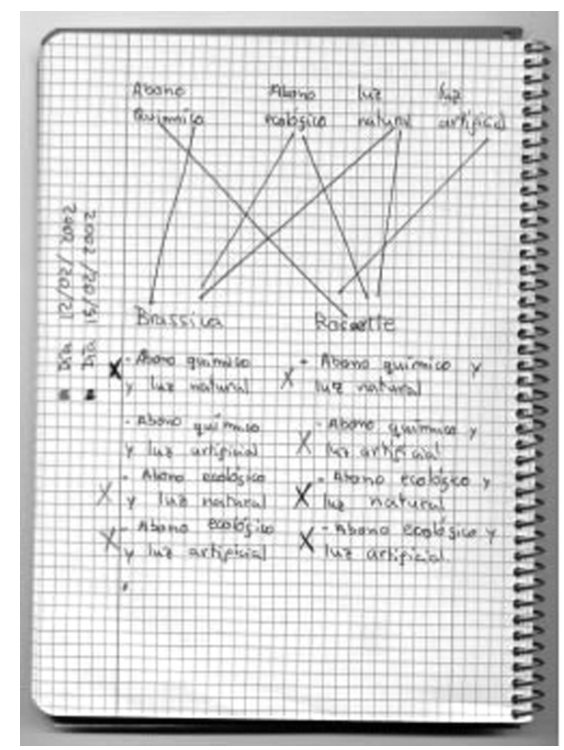

David fue uno de los 12 participantes que completó el espacio problema, es decir, que diseñó las 8 combinaciones de variables posibles en la primera oportunidad. Recordemos que en la primera sesión diseñó BAE, RNQ, BNE, y RAQ. Mientras que en la segunda diseñó: RNO, BNQ, RAO y BAQ. Vemos también en el protocolo verbal cómo explicita la necesidad de utilizar algún tipo de inscripción para apoyar su estrategia experimental (la combinación factorial de variables para cubrir todo el espacio problema.) Los resultados cuantitativos nos mostraron una correlación significativa entre la proporción de notas completas y el porcentaje del espacio problema cubierto, de manera que los alumnos que completaron el espacio problema presentaban un porcentaje de notas completas significativamente mayor que lo que no lo completaron

En la sesión 4, antes de diseñar los dos últimos experimentos (9 y 10) se pidió a los participantes que escribieran un informe sobre lo que habían descubierto mediante su investigación. La figura 5 muestra la hoja intermedia del informe de David en la que se puede observar una estructuración de los datos obtenidos hasta el momento en forma de doble lista.

$\mathrm{Al}$ preguntarle qué experimentos quería diseñar en la $4^{\mathrm{a}}$ sesión, David respondió con una conciencia clara de lo que le quedaba por hacer, indicando una buena comprensión de la tarea:

Tengo que repetir alguno. No tengo nada nuevo por hacer. No tengo más posibilidades (combinaciones). Tengo que repetir. Voy a repetir uno con Rosette porque sólo be tenido una que ha crecido con Rosette, y aquí hay otra que aún tiene que crecer. Voy a ver si crece.

Vemos que David utiliza con éxito la estrategia experimental de combinación factorial de variables, apoyándose en la notación para poder ser efectivo. Si nuestro diseño hubiera consistido en un análisis de las estrategias de combinación factorial David habría obtenido la máxima puntuación. Sin embargo, esta capacidad mostrada por David no va acompañada de una conciencia del significado de la combinación factorial de variables en tareas investigadoras. David no es consciente de la necesidad de controlar variables para poder determinar inequí- 
FIGURA 5

Página central del Informe (Sesión 4)

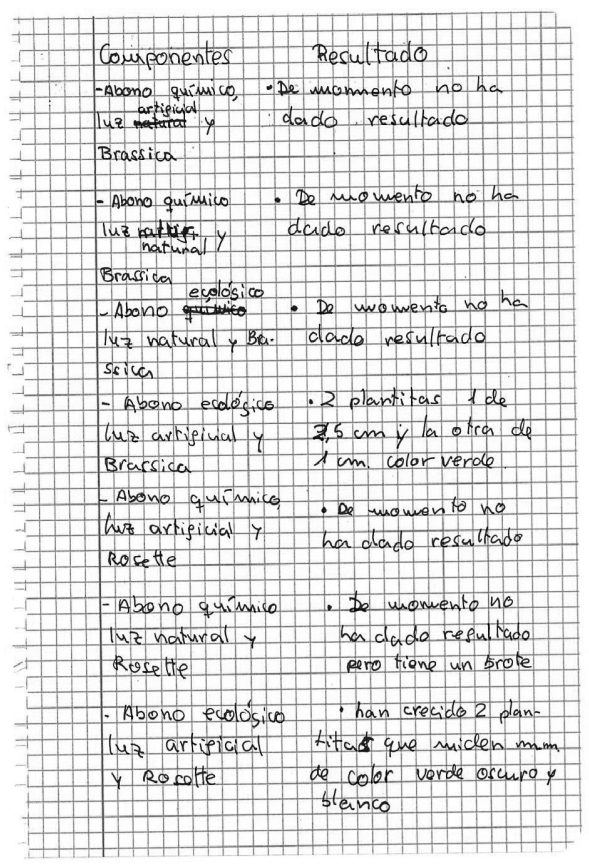

vocamente la causa de determinados efectos. El diseño microgenético así como el hecho de tomar como objeto de análisis las estrategias implicadas en el ciclo completo de la investigación nos permite poner de manifiesto la incoherencia entre la pericia de David al obtener una base de datos óptima sobre la cual hacer inferencias basadas en la base de datos completa y controlada, y la falta de reconocimiento de la utilidad de dichos datos. Veamos en el siguiente apartado esta falta de conciencia de David sobre la necesidad de diseñar experimentos controlados para poder hacer inferencias válidas.

\section{Estrategia basada en el control de variables}

Los datos cuantitativos muestran que de los 34 participantes, sólo 13 mostraron algún uso de esta estrategia de manera más o menos explícita. Paradójicamente, estos no coincidían con los participantes que aplicaron con éxito la estrategia de combinación factorial. En este grupo es donde se sitúa David. Aquí volvimos a relacionar la proporción de notas completas con la aplicación de las estrategias de control de variables y los resultados nos mostraron nuevamente una correlación significativa.

El análisis cualitativo muestra que David obtuvo una base de datos con todos los experimentos controlados sin ninguna intención explícita, sólo como consecuencia de haber completado el espacio problema, y con un registro doblemente escrito, por un lado en sus observaciones on-line (Holmes, 1987) y por el otro, al realizar la doble lista en el informe intermedio.

En el desarrollo de la entrevista nos muestra la falta de conciencia de la necesidad de controlar variables en dos momentos distintos. El primero corresponde a nuestra pregunta sobre lo que pretende averiguar con el experimento en concreto. El segundo momento corresponde a la pregunta nues- 
tra de lo que ha averiguado con el experimento en cuestión. En ninguno de los experimentos diseñados por David, éste respondió que quería averiguar la diferencia entre los resultados generados por un experimento controlado (fruto de la estrategia de control de variables). A la pregunta: ¿Qué pretendes averiguar con este experimento? David normalmente contestaba: Ver lo que pasa. Ocasionalmente respondería de manera más concreta (ver en la sesión1), después de diseñar BAE (exp. 1) y RNC (exp. 2), David contestaba que quería averiguar qué pasaba con los tres factores. Ya hemos destacado la estrategia de cambiar todas la variables a la vez (Tschirgi, 1980) para ver si el efecto se magnifica, contraponiendo todo lo que "yo creo es lo mejor" con "todo lo que yo creo es lo peor". En cambio, en el tercer experimento (BNE), a pesar de ser un control para el factor luz junto con el primer experimento (BAE), no parece que David sea consciente de ello ya que contesta:

\section{Este (BNE) crecerá mejor porque es todo natural, es más como la naturaleza}

En el siguiente experimento $\left(4^{\circ}\right)$, insiste en su teoría de la natural mejor, y para ponerla a prueba vuelve a diseñar un experimento con todo lo opuesto "a lo natural" (RAC) y predice: No crecerá tanto como todo natural. Es interesante observar cómo un uso destacado de la estrategia de combinación factorial no va acompañada de una conciencia de la necesidad de tener una base de datos con experimentos controlados, i/o el espacio problema completo para hacer inferencias.

\section{Estrategias Inferenciales: interpretación de los datos}

Un segundo momento en el que se pudo mostrar esta toma de conciencia es cuando los participantes observaban sus plantas y se les pedía que explicaran sus resultados. Cuando David en la sesión 3 dio cuenta de sus resultados, dijo:

la número 3 (número asignado en su libreta de notas y usado de manera consistente) ha crecido, mira, porque ésta ha crecido (BAE) y las otras no (...). BAE ha crecido más que las otras (...) creo que la Brassica es buena semilla, No sé, porque tú has dicho que los científicos de otro país ya la babían probado. Yo creo que (Brassica) crecerá, y con (luz) artificial crece bien, porque hay algunas plantas que necesitan luz natural y algunas luz artificial (vemos en su comentario un indicio de refutación de su teoría de "lo natural mejor", combinado con lo que parece una protección de su teoría previa, algo por otro lado muy común según la literatura (Kuhn et al., 1995).

David no acompañó ninguna de las observaciones apelando a las comparaciones controladas (podía comparar los resultados de BAE vs. BNE o los de $\mathrm{BAE}$ vs. RAE, a pesar de que los anotó todos rigurosamente en su libreta. En la sesión 4 hizo inferencias del mismo tipo que en la sesión 3. Observamos en las figuras 2 y $3^{2}$, las anotaciones correspondientes a les sesiones 2,3 y 4 en los colores correspondientes, así como en la doble lista del registro escrito.

Esta incoherencia entre la rigurosidad de las notaciones y la falta de inferencias válidas basadas en experimentos controlados resulta bien explicada por nuestros datos cuantitativos (Garcia-Mila et al., 2009) según los cuales no se observó ninguna relación significativa entre la práctica inscripcional per se, y el uso de estrategias inferenciales válidas. Sin embargo esta correlación resultó estadísticamente significativa cuando se analizaron sólo los participantes que revisaban sus notas. Por tanto, en la línea de Klein observamos que para hacer inferencias válidas no es suficiente con anotar, sino que es necesario revisar lo que se anota. 
Los resultados de David, así como sus anotaciones, apuntaban hacia dos posibles inferencias, una para la luz y la otra para la semilla. Sin embargo David ignoró los datos obtenidos y se limitó a hacer inferencias que confirmaban sus teorías previas. En las sesiones 6 y 7 observamos un cierto progreso que iría de inferencias basadas en su teoría, a una inferencia de tipo generalizada, basada en todos los datos obtenidos, necesariamente utilizando su registro escrito, pues en la sesiones 6 y 7 los experimentos de la primera sesión ya se habían desechado.

Tengo más plantas que crecen más altas con luz artificial que con luz natural (?) Porque la luz natural puede ser que le dé algo que necesite para crecer, algo que la luz natural no le da (...) Porque de todas las plantas que tengo, las que tienen Brassica crecen más altas que las de Rosette, y tengo más plantas con luz artificial que con luz natural que han crecido.

En sus conclusiones, David afirma: He descubierto que las plantas con luz natural han crecido, pero no tanto. Yo creo que con luz artificial crecen mejor que con luz natural.

Vemos en el fragmento final como David es capaz de hacer inferencias refutativas de tipo generalizado, basado en la base de datos general, y entendemos que hay un uso implícito del registro escrito pues los experimentos a los que se refieren ya habían sido desechados.

\section{DISCUSIÓN}

El análisis de David, apoyado mediante referencias puntuales a los resultados cuantitativos publicados en Garcia-Mila et al. (2009), nos ha mostrado la estrecha relación entre las estrategias de investigación empírica y las prácticas inscripcionales dando cuenta del segundo y tercer objetivos del presente trabajo. Las escasas investigaciones publicadas sobre este tema muestran que los alumnos de enseñanza primaria no hacen inscripciones de manera espontánea cuando se les pide que investiguen un problema concreto (Garcia-Mila y Andersen, 2007; Keys, 1999; Klein, 2000), a pesar de que cuando las hacen, se benefician claramente de ello (Lehrer y Schauble, 2006; Lehrer et al., 2000).

David es un buen ejemplo de regulación mutua entre las inscripciones y la organización de la recogida de datos. Esta regulación ha puesto de manifiesto las tres funciones descritas en la introducción. En primer lugar hemos podido observar cómo las notas de David cumplen una función organizativa para aplicar las estrategias de diseño experimental. Primero con la preparación del espacio gráfico, organizando, revisando y corrigiendo la estructuración de las páginas de su libreta como un paso previo para ir registrando sus observaciones. Después con la elaboración de códigos para conseguir registros económicos mientras investigaba, con un sólo encabezado con las variables para los distintos días, y distintos colores para las correspondientes observaciones. También en relación a la función organizativa, hemos visto como la estructuración de los registros en forma de listas (en las notas) o dobles listas en el informe ha permitido "congelar" las observaciones y las ha podido transformar en objeto de conocimiento (Lehrer et al., 2000; Olson, 1994; Siegler y Liebert, 1975), ayudando a David a hacer más visibles aquellos datos discordantes y que según la literatura son más susceptibles de ser obviados y olvidados.

También hemos podido observar la función epistémica (Lemke, 2002). David en la segunda sesión se da cuenta de que para diseñar todas las combinaciones posibles de variables necesita apoyarse no sólo de un registro escrito, sino de un diagrama que le resuelva el problema de combinación factorial, y elabora el diagrama de la Figura 4, que además "traduce" justo debajo 
de él, en forma de lista de experimentos que debe diseñar indicando una buena conciencia de los objetivos de la tarea (metatarea) y las herramientas cognitivas útiles para resolverla. Finalmente, aunque de manera menos explícita, también observamos en la manera de trabajar de David, la función mnemónica de las inscripciones, en la sesiones 6 y 7, cuando ya se han desechado los cuatro experimentos, su referencia a estos resultados para refutar su teoría fuertemente arraigada de "lo natural mejor que lo artificial".

En relación al segundo objetivo, vemos que el análisis microgenético ligado al hecho de que la resolución de la tarea implicaba poner en juego las estrategias del ciclo completo de la investigación científica, nos ha permitido poner de manifiesto no sólo el irregular avance estratégico sino también la aplicación desigual de las estrategias anteriores. David, por ejemplo, muestra ser muy eficiente en el diseño factorial, pero no muestra ninguna intencionalidad en los protocolos verbales de la necesidad de diseñar todos los pares de experimentos controlados posibles para hacer todas las inferencias válidas posibles. Tampoco nos muestra en su manera de proceder ninguna conciencia del requisito de basar las inferencias en experimentos controlados para que sean válidas. Muestra cierto progreso, en lo que de forma implícita se podría atribuir a sus claros registros escritos, haciendo inferencias basadas en la generalidad de los datos. Así pues, el análisis microgenético nos ha permitido ver la estrecha relación de regulación mutua entre el desarrollo de las estrategias de investigación y las prácticas inscripcionales. A nuestro entender, sin embargo, la principal aportación de análisis microgenético es hacer plausible la interpretación metacognitiva de dicha regulación. Según Lehrer y Schauble (2000), más que la adquisición de un gran repertorio de herramientas gráficas, lo importante es reconocer y comprender la función de las inscripciones. Este reconocimiento lo hemos visto cuando David recurre a la estructuración del espacio gráfico, o al diagrama para resolver la tarea del diseño experimental, con un reconocimiento de la utilidad del diagrama para resolver su tarea concreta, mostrando el componente metatarea de la metacognición (Kuhn, 2002). Finalmente, y aunque sea no sea un gran progreso, vemos el efecto regulador implícito del registro escrito y su revisión en el abandono y refutación de su teoría de luz natural mejor que la luz artificial. Este progreso necesariamente pasaría por el reconocimiento del valor del registro escrito y de la incoherencia entre el registro escrito y su teoría previa, en la línea de la cita de Holmes, el registro escrito sería un medio para encontrar las incoherencias.

Finalmente queremos hacer mención a dos características que presenta la tarea planteada en este trabajo en tanto que facilitadora de la conciencia de la necesidad y de los beneficios de las prácticas inscripcionales mientras se investiga. El hecho de que la tarea implicara que el alumno tuviera que esperar para observar el crecimiento y no pudiera hacerlo el mismo día que el diseño, enfatizó la necesidad de tomar notas on-line sobre qué variables ya se habían diseñado y qué resultados se habían obtenido, y a su vez una necesidad de revisarlas. También el hecho de que el ciclo de crecimiento durara dos semanas obligó a los alumnos a recoger datos de manera iterativa en lugar de manera puntual en una sola sesión. De esta forma sus conclusiones tenían que basarse en los datos acumulados, y deseablemente registrados en las diversas sesiones. La iteración en la secuenciación de la tarea es una característica que ya proponen Wu y Krajcik (2006) en su trabajo sobre tablas y gráficos como ayuda para la investigación. Por otro lado, la idea de recogida de datos y observación de éstos en días diferentes está recogida por Lehrer $e t$ al. (2000) bajo el concepto de historicidad. Estos autores subrayan la impor- 
tancia de la historia de las inscripciones para la investigación y el aprendizaje en el aula de ciencias en un doble sentido. El primero se refiere a la historia de la propia inscripción. El hecho de que las producciones inscripcionales vayan evolucionando, se vayan adaptando a la tarea, se revisen, se editen, se reestructuren, y dimensionalicen hace, según los autores, que potencialmente tengan un futuro en el repertorio del alumno, de forma que la estrategia inscripcional se incorpore como estrategia útil en futuros proyectos. El otro sentido del concepto al que estos autores aluden hace referencia a la idea que preserva historia. Es decir, sirven no sólo para recuperar lo inscrito, sino también los cambios en las inscripciones. El diseño de la tarea tal como la hemos presentado a los alumnos en este estudio cumple las dos características anteriores y de manera comparativa con estudios anteriores, ha dado resultados mucho más prometedores en el sentido de promover la toma de notas on-line y facilitar la regulación mutua entre las prácticas inscripcionales y la investigación científica en alumnos de final de enseñanza primaria (Garcia-Mila et al., 2009). Estos dos aspectos deben de tenerse en cuenta en el diseño de actividades de aprendizaje en las aulas de ciencias para promover el aprendizaje, el desarrollo de las estrategias investigadoras y las prácticas inscripcionales.

\section{Notas}

${ }^{1}$ Falta la línea que conecta Brassica con luz artificial

${ }^{2}$ En las sesions $2^{\mathrm{a}}$ y $4^{\mathrm{a}}$ David hace las anotaciones correspondientes, de manera muy similar a las de las figuras 2 y 3.

\section{Referencias}

CHeng, P. \& Novick, L. (1992). Covariation in natural causal induction. Psychological Review, 99, 365-382.

Duschl, R. A., SCHWEINGRuber, H. A. \& SHOSE, A. W. (Eds.) (2007). Taking science to school: Learning and teaching science in Grades K-8 Committee on Science Learning, Kindergarten through Eighth Grade. Washington, DC: The National Academies Press.

Eberbach, C. \& Crowley, K. (2009). From everyday to scientific observation: How children learn to observe in the biologist's world. Review of Educational Research, 79 (1), 39-68.

ForD, D. (2005). The challenges of observing geologically: Third graders' descriptions of rock and mineral properties. Science Education, 89, 276-295.

Garcia-Mila, M. \& ANDERSEN, C. (2007). Developmental change in note-taking during scientific inquiry. International Journal of Science Education, 29, 1035-1058.

Garcia-Mila, M., Andersen, C. \& Rojo, N. (2009). Representational practices and scientific inquiry En C. Andersen, N. Scheuer, M. P. P. Echeverría \& E. Teubal (Eds.), Representational systems and practices as learning tools (pp. 167-185). Rotterdam: Sense Publishers.

Gruber, H. (1974). Darwin on man: A psychological study of scientific creativity. Chicago: University of Chicago Press.

HOLMES, F. L. (1987). Scientific writing and scientific discovery. Isis, 78, 220-235.

KANARI, Z. \& MillaR, R. (2004). Reasoning from data: how students collect and interpret data in scientific investigations. Journal of Research in Science Teaching, 41, 748-769.

KARMILOFF-Smith, A. (1979). Micro- and macrodevelopmental changes in language acqusition and other representational systems. Cognitive Science, 3, 91-118.

KARMILOFF-SMITH, A. (1992). Beyond modularity. A developmental perspective on cognitive science. Cambridge, MA: The MIT Press

KeYs, C. W., Hand, B., Prain, V. \& Collins, S. (1999). Using the science writing heuristic as a tool for learning from laboratory investigations in secondary school. Journal of Research in Science Teaching, 36, 1065-1084.

KLACZYNSKI, P. (2000). Motivated scientific reasoning biases, epistemological beliefs, and theory polarization: A two process approach to adolescent cognition. Child Development, 71, 1347-1366.

KLAHR, D. (2000). Exploring science: The cognition and development of discovery processes. Cambridge, MA: The MIT Press.

KLEIN, P. D. (2000). Elementary students' strategies for writing-to-learn in science. Cognition and Instruction, 18, 317-348.

KuHN, D. (2002). A multi-component system that constructs knowledge: Insights from microgenetic study. En N. Granott \& J. Parziale (Eds.), Microdevelopment: Transition processes in development and learning (pp. 109-131). Cambridge, UK: Cambridge University Press

Kuhn, D., Garcia-Mila, M., Zohar, A. \& Andersen, C. (1995). Strategies of knowledge acquisition. Monograph of the Society for Research in Child Development, 60 (4), Serial No. 245.

Latour, B. (1990). Drawing things together. En M. Lynch \& S. Woolgar (Eds.), Representation in scientific practice (pp. 19-68). Cambridge, MA: MIT Press.

Latour, B. \& WoOlgar, S. (1979). Laboratory life: The construction of scientific facts. Princeton, NJ: Princeton University Press.

Lehrer, R. \& SCHAuble, L. (2000). Modeling in mathematics and science. En R. Glaser (Ed.), Advances in instructional psychology. Educational design and cognitive science (pp. 101-159). Mahwah, NJ: Lawrence Erlbaum. 
Lehrer, R., \& Schauble, L. (2006). Scientific thinking and science literacy. En W. Damon, R. Lerner, K. Anne Renninger \& I. E. Sigel (Eds,), Handbook of child psychology: Vol. 4. Child psychology in practice (6a ed., pp. 153-196). Hoboken, NJ: Wiley.

Lehrer, R., Schauble, L., Carpenter, S. \& Penner, D. (2000). The interrelated development of inscriptions and conceptual understanding. En P. Cobb, E. Yackel \& K. McClain (Eds.), Symbolizing and communicating in mathematics classrooms. Perspectives on discourse, tools and instructional design (pp. 325-360). Mahwah, NJ: Lawrence Erlbaum.

LEMKE, J. (2002). Enseñar todos los lenguajes de la ciencia: Palabras, símbolos, imágenes y acciones. En M. Benlloch (Ed.), La educación en ciencias. Ideas para mejorar su práctica (pp. 159-186). Barcelona: Paidós.

MASNICK, A. M. \& KLAHR, D. (2003). Error matters: An initial exploration of elementary school children's understanding of experimental error. Journal of Cognition and Development, 4 (1), 67-98.

OLson, R. D. (1994). The world on paper. Cambridge: Cambridge University Press.

SCHAUBLE, L. (1990). Belief revision in children: The role of prior knowledge and strategies for generating evidence. Journal of Experimental Cbild Psychology, 49, 31-57.

SiegleR, R. S. \& LIEBERT, D. E. (1975). Acquisition of formal scientific reasoning by 10- and 13-year-olds: Designing a factorial experiment. Developmental Psychology, 11, 401-402.

TsCHIRGI, J. E. (1980). Sensible reasoning: A hypothesis about hypotheses. Child Development, 51, 1-10.

Twenty, R. D. (1991). Faraday's notebooks: The active organization of creative science. Physics Education, 26, 301-306.

WeLLS, G. (1999). Dialogic inquiry. Cambridge, UK: The Press Syndicate of the University of Cambridge.

WU, H.-K. \& KRAJCIK, J. S. (2006). Inscriptional practices in two inquiry-based classrooms: A case study of seventh graders' use of data tables and graphs. Journal of Research in Science Teaching, 43, 63-95.

ZimMERMAN, C. (2000). The development of scientific reasoning skills. Developmental Review, 20, 99-149.

ZIMMERMAN, C. (2005). The development of scientific reasoning skills: What psychologists contribute to an understanding of elementary science learning. Washington, DC: National Research Council. 